\title{
7 Die Regionalisierung der Investitionen und kaufkraftrelevanten Einkommen
}

Neben den demographischen Effekten, die durch die Studierenden an den Wiener Hochschulen sowie deren Rolle als Arbeitgeber beeinflusst werden, sind die regional wirksamen ökonomischen bzw. monetären Wertschöpfungseffekte für diese Studie von zentraler Bedeutung. Diese umfassen drei Aspekte: erstens die Einkommen der Universitätsangestellten, zweitens die Ausgaben der Studierenden und drittens die Bau-, Investitions- und Sachausgaben der Hochschulen. Um diese für die Stadt Wien plausibel abschätzen zu können, ist vorab eine Regionalisierung der Ausgaben sowie der Investitionen bzw. Sachausgaben notwendig, die anschließend im Hinblick auf ihre regionalwirtschaftlichen Investitions- und Arbeitsmarkteffekte untersucht werden.

\subsection{Die Ausgaben der Studierenden}

Um die Ausgaben der Studierenden zu schätzen, wird auf die Ergebnisse der Studierendensozialerhebung zurückgegriffen, die 2009 und 2011 vom Institut für höhere Studien durchgeführt wurden (vgl. Unger et al. 2010, Unger et al. 2012). Demnach betrugen die Ausgaben der Studierenden in Österreich im Jahr 2011 durchschnittlich 930,9 Euro pro Monat, 2009 lagen diese Ausgaben noch bei 902 Euro. Da die Daten 2011 nicht mehr wie 2009 nach Bundesländern differenziert worden sind, wurden die wienbezogenen Daten von 2009 um den Anstieg der österreichweiten Ausgaben in den jeweiligen Ausgabengruppen gewichtet, womit für Wien ein durchschnittlicher Anstieg der Ausgaben unterstellt wird. Demnach lagen im Jahr 2011 die geschätzten monatlichen Ausgaben bei 942,1 Euro pro Studierenden in Wien (Tabelle 7.1). Die beiden größten Ausgabenposten der Studierenden entfallen auf die Bereiche Wohnen und Ernährung, die am Studienort Wien 54,5 \% der Ausgaben ausmachen - immerhin 4,1 \% mehr als bei den österreichischen Studierenden insgesamt.

Bei der Schätzung der wertschöpfungsrelevanten Ausgaben der Studierenden an den Wiener Hochschulen wurden folgende Umstände berücksichtigt:

- erstens weisen die Studierenden an den Wiener Hochschulen eine sehr ungleiche Geschlechterstruktur auf, der Frauenanteil liegt bei 53,1 \% (Kapitel 5.4),

- zweitens existieren beträchtliche Kaufkraftströme zwischen Wien und dem Wiener Umland, die berücksichtigt werden müssen, und

- drittens sind 14,9\% der Studierenden Tagespendler, womit von einem anderen Ausgabenverhalten auszugehen ist als bei den in Wien lebenden Studierenden.

Eine Differenzierung der Ausgaben nach Frauen und Männern macht deshalb Sinn, da diese 2011 deutlich, um insgesamt 9,1\%, vom Durchschnittswert abweichen: Weibliche Studierende geben im Durchschnitt 4,2\% weniger aus, männliche Studierende 4,9 \% mehr (Unger et al. 2011, 313). Angesichts der existierenden Geschlechterpropor- 
Tabelle 7.1: Monatliche Ausgaben der Studierenden in Wien und Österreich 2011 nach Ausgabenarten

\begin{tabular}{lrrrr}
\hline & \multicolumn{2}{c}{ Österreich* } & \multicolumn{2}{c}{ Wien** } \\
& Euro & in $\%$ & Euro & in \% \\
\hline Wohnen & 301,7 & 32,4 & 314,7 & 33,4 \\
Ernährung & 191,8 & 20,6 & 199,2 & 21,1 \\
Kleidung & 50 & 5,4 & 49,5 & 5,3 \\
Mobilität & 74,2 & 8,0 & 63,8 & 6,8 \\
Kommunikation & 31,3 & 3,4 & 32,6 & 3,5 \\
Freizeit & 88,8 & 9,5 & 91,1 & 9,7 \\
Studium & 77,7 & 8,3 & 76,4 & 8,1 \\
Sonstiges & 115,5 & 12,4 & 114,8 & 12,2 \\
\hline Gesamt & $\mathbf{9 3 0 , 9}$ & $\mathbf{1 0 0 , 0}$ & $\mathbf{9 4 2 , 1}$ & $\mathbf{1 0 0 , 0}$ \\
\hline
\end{tabular}

*) Daten: Studierendensozialerhebung 2011

**) Grundlage: Daten der Studierendensozialerhebung 2009, gewichtet um den Anstieg der Werte für Österreich zwischen 2009 und 2011

Quellen: Unger et al., 2010; Unger et al., 2012, Studierendensozialerhebung.

tionen ist diese getrennte Berechnung der Ausgaben sinnvoll. Zu diesem Zweck wurde das Verhältnis der männlichen und weiblichen Studierenden für Österreich auf die Studierenden in Wien übertragen. Diese Schätzung wurde mit der Anzahl der Studierenden multipliziert und ergibt in Summe einen geschätzten Betrag von 174,2 Mio. Euro, den die Studierenden der Wiener Hochschulen monatlich ausgeben. Dieser Schätzung liegt die Annahme zugrunde, dass die Ausgaben der Studierenden in Wien an allen Universitäten gleich sind.

Für die regionalökonomische Wirksamkeit der Haushaltsausgaben - insbesondere im Bereich des Konsums - stellen die Kaufkraftflüsse eine wichtige Größe dar (vgl. Doubek et al. 1993, 77). Die Schätzung der Wertschöpfungseffekte, die sich durch

Tabelle 7.2: Monatliche Ausgaben der Studierenden an Wiener Hochschulen 2011 unter Berücksichtigung der Geschlechterrelation

\begin{tabular}{|c|c|c|c|c|c|c|}
\hline & \multicolumn{2}{|c|}{ Österreich } & \multicolumn{2}{|r|}{ Wien } & \multirow{2}{*}{$\begin{array}{c}\text { Anzahl } \\
\text { Studierende }\end{array}$} & \multirow{2}{*}{$\begin{array}{c}\text { Ausgaben } \\
\text { Studierende Wiens }\end{array}$} \\
\hline & Euro & Gewichtungsf. & Euro & Schätzung & & \\
\hline Ausgaben gesamt & 930,9 & & 942,1 & & & 174.238.119 \\
\hline Frauen & 892,0 & 0,958 & & 902,7 & 98.105 & 88.562 .435 \\
\hline Männer & 977,0 & 1,049 & & 988,8 & 86.650 & 85.675 .684 \\
\hline
\end{tabular}

Quellen: eigene Erhebung 2013; Uni-data 2013; Unger et al., 2010; Unger et al., 2012;

Studierendensozialerhebung; eigene Berechnungen. 
die Ausgaben der Studierenden für die Stadt Wien ergeben, ist weniger mit der Frage der Verschiebung von Kaufkraft zwischen Bezirken und Geschäftsstraßen innerhalb Wiens, sondern der Verlagerung in das Wiener Umland verbunden. 1970 flossen noch 3,0\% der Wiener Kaufkraft in das Umland ab, 1990 - in der letzten von der Stadt Wien durchgeführten Kaufkraftanalyse - bereits 11,7\% (Doubek et al. 1993, 82). In einer jüngeren Studie der Wirtschaftskammer Wien wurden die Kaufkraftabflüsse von Wien in das Wiener Umland mit 8,6 \% angegeben (WK Wien/CIMA 2007, 43). Kaufkraftabflüsse in andere Bundesländer und in das Ausland werden - aus Gründen der geringen Mobilität von Studierenden - nicht berücksichtigt.

Für die 2011 in Wien lebenden Studierenden (157.359) wird bei den kaufkraftabflussrelevanten Ausgabenposten der durchschnittliche Wert von $-8,6 \%$ berücksichtigt (vgl. Tabelle 7.3). Die Ausgaben für Wohnen und Studium sind an den Standort Wien gebunden, daher werden hier keine Kaufkraftabflüsse geltend gemacht. Für die in Wien lebenden Studierenden reduzieren sich die (um die Kaufkraftabflüsse) bereinigten Ausgaben auf 894,7 Euro monatlich. Ebenso wird für die im Wiener Umland lebenden Studierenden der Kaufkraftzufluss nach Wien geschätzt, wobei hier auf keine

Tabelle 7.3: Bereinigte monatliche Gesamtausgaben der Studierenden Wiens 2011

\begin{tabular}{|c|c|c|c|c|c|}
\hline & \multirow[b]{2}{*}{ Ausgaben } & \multicolumn{2}{|c|}{ Wien } & \multicolumn{2}{|c|}{ Umland } \\
\hline & & $\begin{array}{c}\text { KK-Abfluss } \\
(\%)\end{array}$ & $\begin{array}{c}\text { Ausgaben } \\
\text { bereinigt }\end{array}$ & $\begin{array}{c}\text { KK-Abfluss } \\
(\%)\end{array}$ & $\begin{array}{c}\text { Ausgaben } \\
\text { bereinigt }\end{array}$ \\
\hline kk-relevante Ausgaben & 551,0 & & & & \\
\hline Ernährung & 199,2 & $-8,6$ & 182,1 & 23,4 & 46,6 \\
\hline Kleidung & 49,5 & $-8,6$ & 45,2 & 23,4 & 11,6 \\
\hline Mobilität & 63,8 & $-8,6$ & 58,3 & 23,4 & 14,9 \\
\hline Kommunikation & 32,6 & $-8,6$ & 29,8 & 23,4 & 7,6 \\
\hline Freizeit & 91,1 & $-8,6$ & 83,3 & 23,4 & 21,3 \\
\hline Sonstiges & 114,8 & $-8,6$ & 104,9 & 23,4 & 26,9 \\
\hline nicht kk-relevante Ausgaben & 391,1 & & & & \\
\hline Wohnen & 314,7 & 0,0 & 314,7 & 0,0 & 0,0 \\
\hline Studium & 76,4 & 0,0 & 76,4 & 100,0 & 76,4 \\
\hline Ausgaben gesamt & 942,1 & & 894,7 & & 205,3 \\
\hline Studierende gesamt (184.755) & 174.064 .435 & & & & \\
\hline Studierende Wien (157.359) & & & 140.796 .595 & & \\
\hline Studierende Umland (27.396) & & & & & 5.624 .806 \\
\hline bereinigte Gesamtausgaben $\mathrm{S}$ & Idierende W & & 146.421.401 & & \\
\hline
\end{tabular}

Quellen: eigene Erhebung 2013; Unger et al., 2010, Studierendensozialerhebung; WK Wien/ Cima, 2007; WK NÖ/Cima, 2006; eigene Berechnungen. 
Erfahrungen bzw. Vergleichsdaten aus anderen Studien zurückgegriffen werden kann. Ansatz für die Schätzung ist eine Berechnung der Kaufkraftabflüsse der niederösterreichischen Haushalte nach Wien, die für das Jahr 2006 mit 11,7 \% berechnet wurden (WK NÖ/CIMA 2006, 32). Da es sich bei Studierenden um keine durchschnittlichen Haushalte handelt und diese überdies täglich nach Wien pendeln, wurde dieser Wert für kaufkraftabflussrelevante Ausgaben verdoppelt $(23,4 \%)$ sowie die auf das Studium bezogenen Ausgaben zu 100,0 \% als Kaufkraftzufluss gewertet. Damit ergibt sich für die 27.396 im Wiener Umland lebenden Studierenden ein Kaufkraftzufluss von rund 5,6 Mio. Euro monatlich. Die bereinigten Gesamtausgaben der Studierenden an Wiener Hochschulen, aus denen sich unmittelbare Wertschöpfungseffekte für die Stadt Wien ergeben, belaufen sich damit monatlich auf 146,42 Mio. Euro bzw. für das gesamte Jahr auf 1.757,06 Mio. Euro.

\subsection{Die Einkommen der Universitätsangestellten}

Die Ausgaben für das Personal stellen für Hochschulen den größten Ausgabenposten dar: Im Jahr 2011 wurden etwa von der Universität Wien 321,6 Mio. Euro für Personalkosten aufgewendet, das sind 61,4 \% der Gesamtausgaben (Musil 2012). Für die Wiener Hochschulen, die für diesen Punkt Daten zur Verfügung gestellt haben, beliefen sich die Aufwendungen auf 564 Mio. Euro, was einem Großteil der Gesamtausgaben entspricht. Gegenüber dem Vergleichsjahr 2009, in dem die Personalkosten 540 Mio. Euro ausmachten, bedeutet das einen Anstieg von 4,5\%, wobei dieser deutlich unter dem Anstieg der allgemeinen Ausgaben (25,2\%) blieb. Dieser große Ausgabenposten wird für die Schätzung der regionalen Wertschöpfungseffekte nach zwei Kriterien differenziert: erstens nach verschiedenen Bruttoeinkommensklassen, zweitens nach dem Wohnort der Beschäftigten bzw. der regionalen Zuteilung der Nettoausgaben der Hochschulbediensteten.

\section{Die Gehaltsklassen der Beschäftigten der Wiener Hochschulen}

Die Differenzierung der Bruttogehaltsklassen erfolgte für die Beschäftigten nach allgemeinem und wissenschaftlichem Personal. In Summe umfassen die Bruttogehälter der Beschäftigten 690,5 Mio. Euro, davon entfallen 252,1 Mio. Euro auf das allgemeine Personal und 438,4 Mio. Euro auf das wissenschaftliche Personal ${ }^{1}$. Für die Auswertung nach den Gehaltsgruppen wurden sowohl die Kopfzahl der Beschäftigen als auch die Vollzeitäquivalente berücksichtigt. Insbesondere in den niedrigen Gehaltsstufen beim

Die Dienstgeberabgaben sind hier nicht enthalten, darum weichen die Daten von den Personalausgaben ab, die im Gesamtbudget (dort inklusive Dienstgeberabgaben) angeführt sind. 
Tabelle 7.4: Einkommensstruktur des allgemeinen und wissenschaftlichen Personals der Hochschulen Wiens nach Gehaltsklassen

\begin{tabular}{|c|c|c|c|c|c|}
\hline \multicolumn{6}{|c|}{ Allgemeines Personal } \\
\hline & \multicolumn{2}{|c|}{$\begin{array}{l}\text { Summiert nach } \\
\text { Gehaltsklasse }\end{array}$} & \multicolumn{2}{|c|}{ Jahresbruttogehälter } & \multirow{2}{*}{$\begin{array}{c}\begin{array}{c}\text { Jahresnetto- } \\
\text { gehälter }\end{array} \\
\begin{array}{c}\text { pro Kopf } \\
(\text { in } €)\end{array}\end{array}$} \\
\hline & Kopfzahl & VZ-Äquivalente & $\begin{array}{l}\text { Klassensumme } \\
\text { (in } 1.000 €)\end{array}$ & $\begin{array}{l}\text { pro Kopf } \\
\quad(\text { in } €)\end{array}$ & \\
\hline$\leq 1.000$ & 3.034 & 865 & 15.521 & 5.115 & 5.115 \\
\hline$>1.000$ bis 2.000 & 2.772 & 1.951 & 49.770 & 17.958 & 14.719 \\
\hline$>2.000$ bis 3.000 & 2.979 & 2.794 & 87.653 & 29.424 & 20.865 \\
\hline$>3.000$ bis 4.000 & 1.264 & 1.220 & 53.992 & 42.711 & 27.948 \\
\hline$>4.000$ bis 5.000 & 381 & 375 & 21.069 & 55.246 & 34.342 \\
\hline$>5.000$ bis 6.000 & 158 & 153 & 10.170 & 64.419 & 39.645 \\
\hline$>6.000$ & 143 & 124 & 13.925 & 97.207 & 59.055 \\
\hline \multicolumn{6}{|c|}{ Wissenschaftliches Personal } \\
\hline & \multicolumn{2}{|c|}{$\begin{array}{l}\text { Summiert nach } \\
\text { Gehaltsklasse }\end{array}$} & \multicolumn{2}{|c|}{ Jahresbruttogehälter } & $\begin{array}{c}\text { Jahresnetto- } \\
\text { gehälter }\end{array}$ \\
\hline & Kopfzahl & VZ-Äquivalente & $\begin{array}{l}\text { Klassensumme } \\
\text { (in } 1.000 €)\end{array}$ & $\begin{array}{l}\text { pro Kopf } \\
\text { (in €) }\end{array}$ & $\begin{array}{l}\text { pro Kopf } \\
\text { (in } €)\end{array}$ \\
\hline$\leq 1.000$ & 10.885 & 1.158 & 30.932 & 2.842 & 2.842 \\
\hline$>1.000$ bis 2.000 & 2.831 & 1.436 & 47.815 & 16.888 & 14.108 \\
\hline$>2.000$ bis 3.000 & 2.401 & 1.677 & 62.390 & 25.984 & 18.948 \\
\hline$>3.000$ bis 4.000 & 1.704 & 1.412 & 64.040 & 37.577 & 25.328 \\
\hline$>4.000$ bis 5.000 & 807 & 715 & 40.415 & 50.078 & 31.706 \\
\hline$>5.000$ bis 6.000 & 580 & 543 & 39.367 & 67.925 & 41.822 \\
\hline$>6.000$ & 1.510 & 1.463 & 153.514 & 101.644 & 61.553 \\
\hline
\end{tabular}

Quellen: eigene Erhebung 2013; uni:data 2013; eigene Berechnungen.

wissenschaftlichen Personal ist die Abweichung sehr hoch, obwohl zahlreiche geringfügige Dienstverhältnisse ${ }^{2}$ nicht berücksichtigt worden sind (vgl. Tabelle 7.4).

Die große Zahl an externen Lektoren ist dafür ausschlaggebend, dass in der untersten Gehaltsklasse das Verhältnis zwischen Kopfzahl und Vollzeitäquivalenten $1: 9,4$ ausmacht, bei allen anderen Gehaltsklassen ist dieses unter $1: 2,5$ (beim wissenschaftlichen. Personal) angesiedelt. Für die Bruttogehaltsklassen wurden sowohl die kumulierten Jahresbruttogehälter sowie die durchschnittlichen Jahresbruttogehälter pro Kopf

2 Folgende Dienstverhältnisse wurden in der Auswertung nicht berücksichtigt: Freie Dienstnehmer, Werkvertragsnehmer und Volontäre, Gutachter, beamtetes Lehrpersonal, Fremduniversität sowie Unirat. 
errechnet, die mittels Brutto-Netto-Rechner des Bundesministeriums für Finanzen ${ }^{3}$ in durchschnittliche Jahres- und Monatsnettogehälter umgerechnet wurden. Es handelt sich hier also um die Nettogehälter der durchschnittlichen Bruttogehälter der jeweiligen Gehaltsklasse. Aufgrund der hohen Kopfzahl in der untersten Einkommensklassen des wissenschaftlichen Hochschulpersonals (externe Lektoren, geteilte Prädoc-Stellen) kommt es zu einer gewissen Verzerrung. Aus diesem Grund werden für die Erhebung der regionalen Wertschöpfung die Nettogehälter nach Gehaltsklassen getrennt berücksichtigt.

\section{Regionale Differenzierung und Berücksichtigung von Kaufkraftströmen}

Für die Schätzung jenes Einkommens, das für den Wertschöpfungseffekt auf die Wirtschaft Wiens ausschlaggebend ist, wird ähnlich wie bei den Ausgaben der Studierenden vorgegangen, auch wenn die Datenlage etwas unterschiedlich ist. Dazu wurden folgende Berechnungen durchgeführt:

- Im ersten Schritt wurde das Jahresnettogehalt für jede Einkommensgruppe berechnet und anschließend um jenen Teil des Nettoeinkommens bereinigt, der für Wohnen und Energie ausgegeben wird und daher von Kaufkraftabflüssen nicht betroffen ist. Da nicht wie bei den Studierenden auf eine empirische Befragung (Studierendensozialerhebung) zurückgegriffen werden kann, wurden die Ergebnisse der Konsumerhebung der Statistik Austria berücksichtigt. Gemäß dieser werden von den Äquivalenzeinkommen ${ }^{4}$ der Haushalte 24,6 \% abgezogen, also jener Teil der Ausgaben (Wohnen und Energie), der für Kaufkraftabflüsse nicht relevant ist (Spalte 4 in Tabelle 7.5).

- Im nächsten Schritt wird dieses bereinigte Jahresnettogehalt nach dem Suburbanisierungsgrad (Kapitel 6.2, Tabelle 6.4) regional zugeordnet, wobei angenommen wird, dass diese Verteilung über alle Einkommensklassen gleich ist. Die Summe des Jahresnettogehaltes wird aliquot zu den Köpfen, zu 10,7 \% für das wissenschaftliche und zu 19,3\% für das allgemeine Universitätspersonal, dem Bundesland Niederösterreich zugeordnet (Spalten 5 und 6).

- Nach dieser regionalen Zuteilung wurden abschließend die Kaufkraftzu- und -abflüsse nach bzw. von Wien berücksichtigt (Spalten 7 und 8). Hierbei wurden die gleichen Referenzwerte wie bei den Studierenden angenommen.

Das Ergebnis wird in Spalte 9 dargestellt und ergibt sich aus der Summe des Nettoeinkommens, welches - ceteris paribus - in die Wertschöpfungseffekte der Stadt Wien einfließt. Dieses beläuft sich in Summe, für das allgemeine und wissenschaftliche Hochschulpersonal zusammen (Tabelle 6.5 und Tabelle 6.6), auf 298,9 Mio. Euro

3 vgl. www.bmf.gv.at/Steuern/Berechnungsprogramme/_start.htm (Online Zugriff 12. 8. 2013)

4 Die Haushaltseinkommen wurden um die Personenzahl bereinigt, es handelt sich also um „gewichtete Pro-Kopf-Einkommen bzw. -Ausgaben“ (vgl. Statistik Austria 2012, 21). 
Tabelle 7.5: Berechnung des wertschöpfungsrelevanten Nettoeinkommens des allgemeinen Personals für Wien

\begin{tabular}{|c|c|c|c|c|c|}
\hline & \multicolumn{5}{|c|}{ Allgemeines Personal } \\
\hline & \multirow[t]{2}{*}{1} & \multicolumn{2}{|l|}{2} & 3 & 4 \\
\hline & & \multirow{2}{*}{\multicolumn{2}{|c|}{ pro Kopf (in €) }} & hresnettogehalt & \\
\hline & Kopfzahl & & & Summe & ereinigte Summe* \\
\hline$\leq 1.000$ & 3.034 & \multicolumn{2}{|c|}{5.115} & 5.521 .047 & 11.702 .870 \\
\hline$>1.000$ bis 2.000 & 2.772 & \multicolumn{2}{|c|}{14.719} & 0.794 .198 & 30.758 .825 \\
\hline$>2.000$ bis 3.000 & 2.979 & \multicolumn{2}{|c|}{20.865} & .156 .460 & 46.865 .971 \\
\hline$>3.000$ bis 4.000 & 1.264 & \multicolumn{2}{|c|}{27.948} & .328 .848 & 26.637 .951 \\
\hline$>4.000$ bis 5.000 & 381 & \multicolumn{2}{|c|}{34.342} & 096.880 & 9.875 .048 \\
\hline$>5.000$ bis 6.000 & 158 & \multicolumn{2}{|c|}{39.645} & 258.843 & 4.719 .167 \\
\hline$>6.000$ & 143 & \multicolumn{2}{|c|}{59.055} & .459 .707 & 6.378 .619 \\
\hline \multirow[t]{4}{*}{ Gesamt } & \multicolumn{3}{|l|}{10.732} & \multicolumn{2}{|r|}{136.938 .451} \\
\hline & 5 & 6 & 7 & 8 & 9 \\
\hline & \multicolumn{2}{|c|}{$\begin{array}{l}\text { Jahres-Nettogehalt differenziert } \\
\text { nach Wien/Umland** }\end{array}$} & $\begin{array}{l}\text { KK-Zufluss } \\
\text { nach Wien }\end{array}$ & $\begin{array}{l}\text { KK-Abfluss } \\
\text { aus Wien }\end{array}$ & $\begin{array}{c}\text { Summe des } \\
\text { kaufkraftrele- } \\
\text { vanten Einkom- } \\
\text { mens für Wien }\end{array}$ \\
\hline & Wien & Umland & $(23,4 \%$ von 6$)$ & $(8,6 \%$ von 5$)$ & $(4+8-9)$ \\
\hline$\leq 1.000$ & 9.443 .426 & 2.259 .443 & 528.710 & 812.135 & 9.160 .001 \\
\hline$>1.000$ bis 2.000 & 24.820 .297 & 5.938 .528 & 1.389 .616 & 2.134 .546 & 24.075 .367 \\
\hline$>2.000$ bis 3.000 & 37.817 .677 & 9.048 .294 & 2.117 .301 & 3.252 .320 & 36.682 .658 \\
\hline$>3.000$ bis 4.000 & 21.495 .029 & 5.142 .922 & 1.203 .444 & 1.848 .573 & 20.849 .901 \\
\hline$>4.000$ bis 5.000 & 7.968 .497 & 1.906 .550 & 446.133 & 685.291 & 7.729 .339 \\
\hline$>5.000$ bis 6.000 & 3.808 .050 & 911.118 & 213.202 & 327.492 & 3.693 .759 \\
\hline$>6.000$ & 5.147 .115 & 1.231 .504 & 288.172 & 442.652 & 4.992 .635 \\
\hline Gesamt & & & & & 107.183 .660 \\
\hline
\end{tabular}

*) Gemäß der Wohnortverteilung der Universitätsbediensteten (Tab. 6.4 in Kap. 6.2) leben 10,7 \% des wissenschaftlichen Universitätspersonals und 19,3\% des allgemeinen Universitätspersonals im Wiener Umland. Diesem Anteil entsprechend wurden die Jahresnettogehälter in Spalte 3 auf Spalte 4 und 5 aufgeteilt.

**) Gemäß Konsumerhebung der Statistik Austria werden 24,6 \% der Ausgaben für Wohnen und Energie aufgewendet. Diese wurden in Spalte 6 und 7 von dem Jahresnettogehalt von Wien und Niederösterreich abgezogen.

Quellen: eigene Erhebung 2013; uni:data 2013; eigene Berechnungen. 
Tabelle 7.6: Berechnung des wertschöpfungsrelevanten Nettoeinkommens des wissenschaftlichen Personals für Wien

\begin{tabular}{|c|c|c|c|c|c|}
\hline & \multicolumn{5}{|c|}{ Wissenschaftliches Personal } \\
\hline & \multirow[t]{2}{*}{1} & \multicolumn{2}{|l|}{2} & 3 & 4 \\
\hline & & \multicolumn{4}{|c|}{ Jahresnettogehalt } \\
\hline & Kopfzahl & \multicolumn{2}{|c|}{ pro Kopf (in $€$ ) } & Summe & vereinigte Summe* \\
\hline$\leq 1.000$ & 10.885 & \multicolumn{2}{|c|}{2.842} & 30.933 .973 & 23.324 .216 \\
\hline$>1.000$ bis 2.000 & 2.831 & \multicolumn{2}{|c|}{14.108} & 39.944 .693 & 30.118 .298 \\
\hline$>2.000$ bis 3.000 & 2.401 & \multicolumn{2}{|c|}{18.948} & 45.496 .240 & 34.304 .165 \\
\hline$>3.000$ bis 4.000 & 1.704 & \multicolumn{2}{|c|}{25.328} & 43.165 .487 & 32.546 .777 \\
\hline$>4.000$ bis 5.000 & 807 & \multicolumn{2}{|c|}{31.706} & 25.587 .578 & 19.293.034 \\
\hline$>5.000$ bis 6.000 & 580 & \multicolumn{2}{|c|}{41.822} & 24.238 .510 & 18.275 .837 \\
\hline$>6.000$ & 1.510 & \multicolumn{2}{|c|}{61.553} & 92.963 .521 & 70.094 .495 \\
\hline \multirow[t]{5}{*}{ Gesamt } & 20.718 & & & & 227.956 .822 \\
\hline & 5 & 6 & 7 & 8 & 9 \\
\hline & \multicolumn{2}{|c|}{$\begin{array}{l}\text { Jahresnettogehalt differenziert } \\
\text { nach Wien/Umland** }\end{array}$} & \multirow[t]{2}{*}{$\begin{array}{l}\text { KK-Zufluss } \\
\text { nach Wien }\end{array}$} & \multirow[t]{2}{*}{$\begin{array}{l}\text { KK-Abfluss } \\
\text { aus Wien }\end{array}$} & \multirow{2}{*}{$\begin{array}{c}\text { Summe des } \\
\text { kaufkraftrele- } \\
\text { vanten Einkom- } \\
\text { mens für Wien }\end{array}$} \\
\hline & davon Anteil & davon Anteil & & & \\
\hline & Wien & Umland & $(23,4 \%$ von 6$)$ & $(8,6 \%$ von 5$)$ & $(4+8-9)$ \\
\hline$\leq 1.000$ & 20.828 .329 & 2.495 .887 & 584.038 & 1.791 .236 & 19.621 .130 \\
\hline$>1.000$ bis 2.000 & 26.895 .387 & 3.222 .911 & 754.161 & 2.313 .003 & 25.336 .545 \\
\hline$>2.000$ bis 3.000 & 30.633 .332 & 3.670 .834 & 858.975 & 2.634 .467 & 28.857 .840 \\
\hline$>3.000$ bis 4.000 & 29.063 .999 & 3.482 .778 & 814.970 & 2.499 .504 & 27.379 .465 \\
\hline$>4.000$ bis 5.000 & 17.228 .517 & 2.064 .517 & 483.097 & 1.481 .652 & 16.229 .961 \\
\hline$>5.000$ bis 6.000 & 16.320 .169 & 1.955 .668 & 457.626 & 1.403 .535 & 15.374 .261 \\
\hline$>6.000$ & 62.593 .795 & 7.500 .700 & 1.755 .164 & 5.383 .066 & 58.965 .893 \\
\hline Gesamt & & & & & 191.765 .095 \\
\hline
\end{tabular}

*) Gemäß der Wohnortverteilung der Universitätsbediensteten (Tab. 6.4 in Kap. 6.2) leben 10,7 \% des wissenschaftlichen Universitätspersonals und 19,3\% des allgemeinen Universitätspersonals im Wiener Umland. Diesem Anteil entsprechend wurden die Jahresnettogehälter in Spalte 3 auf Spalte 4 und 5 aufgeteilt.

**) Gemäß Konsumerhebung der Statistik Austria werden 24, 6\% der Ausgaben für Wohnen und Energie aufgewendet. Diese wurden in Spalte 6 und 7 von dem Jahresnettogehalt von Wien und Niederösterreich abgezogen.

Quellen: eigene Erhebung 2013; uni:data 2013; eigene Berechnungen. 
jährlich. Das heißt, über die Nettoeinkommen der an den Wiener Hochschulen tätigen Personen kommt der Stadt Wien in Summe eine zusätzliche Kaufkraft von fast 300 Mio. Euro zugute - abermals ein unzweifelhaft hoher Wert.

\subsection{Bau-, Investitions- und Sachausgaben der Hochschulen}

Die ,allgemeinen“ Ausgaben der Universität umfassen alle Finanzmittel, die nicht für Personalausgaben aufgewendet werden. Im Jahr 2011 beliefen sich die Ausgaben jener Hochschulen, die für die Studie Daten zur Verfügung gestellt haben, auf 353,8 Mio. Euro (vgl. Tabelle 7.7). Seit 2009 sind die allgemeinen Ausgaben sowohl absolut (um rund 71,1 Mio. Euro) als auch prozentuell stärker als die Personalkosten angewachsen. Die allgemeinen Ausgaben umfassen zum Großteil Sachausgaben (231,7 Mio. Euro), darüber hinaus Kosten für die Erhaltung und Instandsetzung von Gebäuden (72,0 Mio. Euro) sowie Investitionen (50,1 Mio. Euro). Ein beträchtlicher Teil der Sachausgaben wird für Gebäudemieten aufgewendet, die etwa im Falle der Universitäten vor allem an die Bundesimmobiliengesellschaft (BIG) gehen. Im Falle der Universität Wien beliefen sich die Aufwendungen für Gebäudemieten im Jahr 2011 auf knapp die Hälfte der Sachausgaben (80,49 Mio. Euro).

Der Wertschöpfungseffekt dieser allgemeinen Ausgaben lässt sich daran ermessen, dass diese im Schnitt zu mehr als zwei Drittel $(68,5 \%)$ in Wien ausgegeben werden, wobei zwischen den einzelnen Hochschulen (vgl. Abbildung 7.1) durchaus beträchtliche Unterschiede bestehen. Diese lassen sich durch eine unterschiedliche Gewichtung der allgemeinen Ausgabengruppen erklären.

Die Regionalisierung dieser allgemeinen Ausgaben erfolgt nach den Standorten der beauftragten Unternehmen, an die die entsprechenden Ausgaben geflossen sind. Dabei zeigt sich, dass diese zu 77,7\% in Österreich investiert worden sind, die restlichen Mittel flossen ins Ausland ab oder sind nicht räumlich zuordenbar (vgl. Tabelle 7.8). Von den in Österreich verorteten Ausgaben der Universität Wien entfällt, wie schon erwähnt, der mit Abstand größte Teil auf Wien (68,5 \%). An zweiter Stelle steht Nie-

Tabelle 7.7: Allgemeine Ausgaben der Hochschulen Wiens 2009 bis 2011

\begin{tabular}{lrrrrrrr}
\hline in Millionen $€$ & \multicolumn{2}{c}{2009} & \multicolumn{2}{c}{2010} & \multicolumn{2}{c}{2011} & \multirow{2}{*}{ Wien 2011 } \\
\cline { 2 - 7 } & Gesamt & Wien & Gesamt & Wien & Gesamt & Wien & Anteil (\%) \\
\hline Bau-/Gebäudeerh. & 43,0 & 39,2 & 44,7 & 40,3 & 72,0 & 43,3 & 60,1 \\
Investitionen & 45,1 & 22,1 & 42,4 & 23,3 & 50,1 & 23,1 & 46,1 \\
Sachausgaben & 194,6 & 166,2 & 197,4 & 165,9 & 231,7 & 175,8 & 75,9 \\
\hline Gesamt & $\mathbf{2 8 2 , 7}$ & $\mathbf{2 2 7 , 5}$ & $\mathbf{2 8 4 , 5}$ & $\mathbf{2 2 9 , 5}$ & $\mathbf{3 5 3 , 8}$ & $\mathbf{2 4 2 , 2}$ & $\mathbf{6 8 , 5}$ \\
\hline
\end{tabular}

Quelle: eigene Erhebung 2013. 
Abbildung 7.1: Regionale Differenzierung der allgemeinen Ausgaben je Hochschule 2011

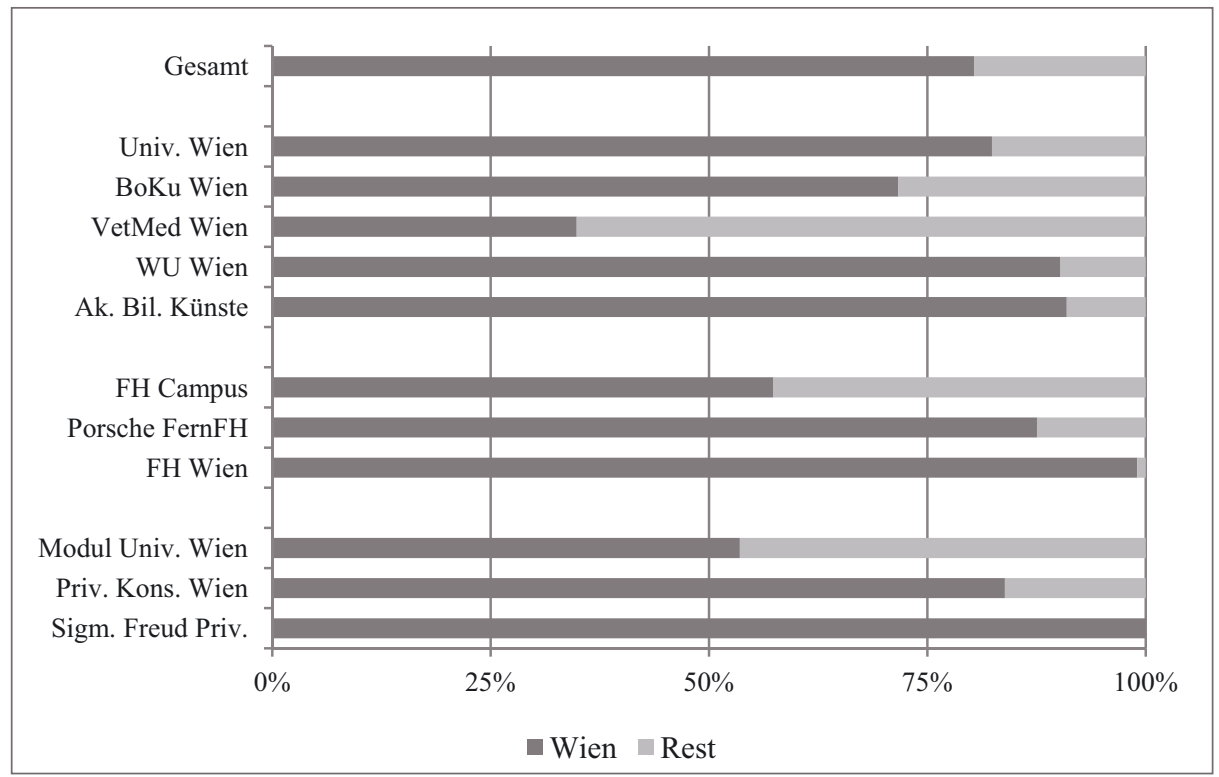

Quellen: eigene Erhebung 2013.

Tabelle 7.8: Regionale Differenzierung der allgemeinen Ausgaben der Hochschulen Wiens $2009-2011$

\begin{tabular}{lrrrc}
\hline in Millionen $€$ & 2009 & 2010 & 2011 & Anteil 2011 (\%) \\
\hline Gesamt & $\mathbf{2 8 2 , 7}$ & $\mathbf{2 8 4 , 5}$ & $\mathbf{3 5 3 , 8}$ & $\mathbf{1 0 0 , 0}$ \\
\hline Ausland & 21,5 & 22,9 & 23,4 & 6,6 \\
Sonstige & 2,4 & 2,8 & 55,6 & 15,7 \\
Österreich & 258,7 & 258,8 & 274,8 & 77,7 \\
\hline Wien & 227,5 & 229,5 & 242,2 & 68,5 \\
Niederösterreich & 15,1 & 14,6 & 16,1 & 4,6 \\
Oberösterreich & 6,1 & 6,7 & 9,3 & 2,6 \\
Burgenland & 4,1 & 2,2 & 1,3 & 0,4 \\
Steiermark & 3,6 & 2,7 & 3,6 & 1,0 \\
Kärnten & 0,3 & 0,3 & 0,3 & 0,1 \\
Salzburg & 1,4 & 1,7 & 1,2 & 0,3 \\
Tirol & 0,5 & 1,0 & 0,7 & 0,2 \\
Vorarlberg & 0,1 & 0,1 & 0,1 & 0,0 \\
\hline
\end{tabular}

Quelle: eigene Erhebung 2013. 
derösterreich mit 4,6 \%, dessen Unternehmen aufgrund der Nähe zu den Standorten der Hochschulen in Wien klar profitieren, weiters gefolgt von Oberösterreich mit 2,6 \%. Auf die restlichen sechs Bundesländer entfallen in Summe gerade 2,1 \% der allgemeinen Ausgaben. Wien und Niederösterreich sind also mit Abstand die größten Profiteure bei den Ausgaben der Universität Wien.

In Summe fließen also der in der Stadt Wien ansässigen Wirtschaft aus den von Wiener Hochschulen getätigten Ausgaben für Baumaßnahmen, Instandhaltung und sonstigen Ausgaben in Geräte und den laufenden Betrieb 242,2 Millionen Euro im Jahr zu. 